\title{
2015 Conant Prize
}

The 2015 Conant Prize was awarded to JeFFREY C. LAGARIAS and CHUANMING Zong at the 121st Annual Meeting of the AMS in San Antonio, Texas, in January 2015.

\section{Citation}

The 2015 Levi L. Conant Prize is awarded to Jeffrey C. Lagarias and Chuanming Zong for their article "Mysteries in packing regular tetrahedra", which appeared in the Notices of the American Mathematical Society 59 (2012), no. 11, 1540-1549.

Finding the best way to pack regular tetrahedra into ordinary three-dimensional space is a problem that goes back to antiquity and remains of great interest today, both in mathematics and materials science. Aristotle claimed regular tetrahedra can fill space with no gaps, but this is false. We still do not know the densest packing, but progress has been rapid in recent years. The article by Jeffrey Lagarias and Chuanming Zong leads the broad range of the Notices readers through the 2,000-year history of the subject, including its appearance in 1900 in Hilbert's eighteenth problem, into its mathematical heart. It uses effective illustrations to highlight surprising discoveries, new techniques, and further conjectures.

\section{Biographical Sketch:}

Jeffrey C. Lagarias was born in Pittsburgh, Pennsylvania, in 1949. He obtained his SB/SM in 1972 and his $\mathrm{PhD}$ in

1974 from the Massachusetts Institute of Technology with a thesis in number theory under the direction of Harold M. Stark. He worked at AT\&T Bell Laboratories

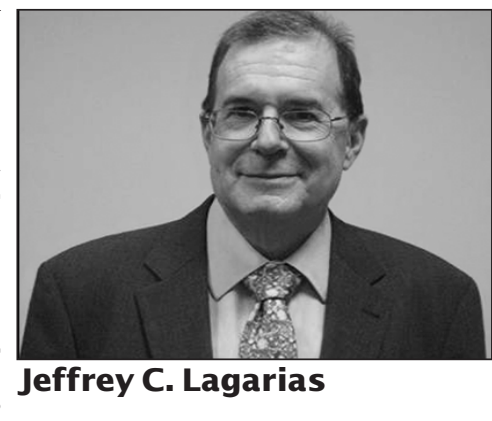
from 1974 to 1995 and at AT\&T Shannon Laboratories from 1995 to 2003. Since that time he has been professor of mathematics at the University of Michigan. He has held visiting positions at the University of Maryland, Rutgers University (computer science), the University of Paris VII (physics), and Stanford University. His research spans many fields, including number theory, optimization, discrete geometry, and computational topology. He has given AMS invited addresses in each of these fields.

DOI: http://dx.doi.org/10.1090/noti1228

\section{Response from Jeffrey C. Lagarias}

I am grateful for receiving the 2015 Levi L. Conant Prize for the Notices of the AMS article with Chuanming Zong. This article was a chance to describe the incredible history of the problem of packing space with congruent regular tetrahedra and to present many attractive and elegant unsolved problems about packing small sets of regular tetrahedra. My $\mathrm{PhD}$ student Elizabeth R. Chen made a significant contribution with her 2008 paper constructing the then densest-known packing, which started a flurry of activity in physics and materials science, leading to tremendous progress on the problem. I am happy that in 2010 she regained, and still holds, the world record for densest packing of congruent regular tetrahedra (jointly with Michael Engel and Sharon C. Glotzer). This problem itself traces back to Aristotle in connection with the physics of the material world. I was interested to discover that Michael Scot, later consigned to the second lowest circle of Hell in Dante's Inferno, and St. Thomas Aquinas, patron saint of colleges and of students, each had connections with the problem. In writing the article, the historical detective work of Dirk Struik, together with an English translation of his Dutch article by Marjorie Senechal, and the expertise of Chuanming were very helpful. Since Beijing is twelve time zones from Ann Arbor, I could work Chuanming Zong on the article all day, email it to Chuanming, who then could also work on it all day! I have received help in learning the skill of writing from many people, including my parents, my advisor Harold M. Stark, and my Bell Labs mentors and colleagues Ron Graham, Andrew Odlyzko, and Ingrid Daubechies, to name only a few. To all of the above, to the AMS, and to the selection committee for the award, I express my deepest gratitude.

\section{Biographical Sketch}

Chuanming Zong was born in Shandong province, China, in 1962. He obtained his B.S. from Shandong University, his MS from the Chinese Academy of Sciences, and his $\mathrm{PhD}$ from Technical University of Vienna. From 1993 to 2000, he worked at the Chinese Academy of Sciences. Since 2000, he has been a professor at Peking University. He was a long-term visitor to IHES (twice), ETH-Zurich, University College London (twice, a research fellow of the Royal Society), TU-Berlin (an Alexander von Humboldt Fellow), MSRI, and others. He works 


\section{American Mathematical Society}

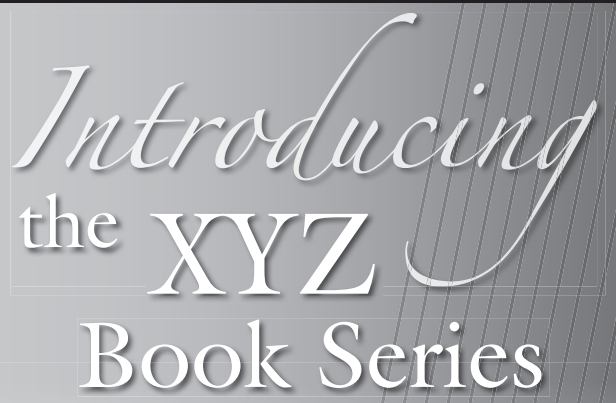

a new distributed publication from the AMS

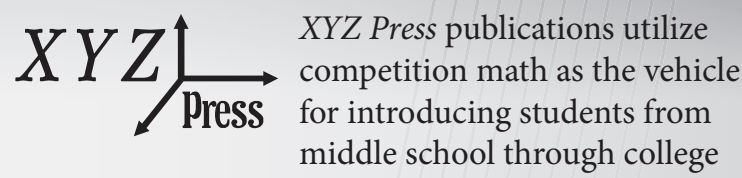

to the elegance of problem-solving through a wide variety of mathematical topics and difficulty levels. Emphasis is placed on developing mathematical insight through interesting problems, many of which employ clever strategies to engage and delight the reader.

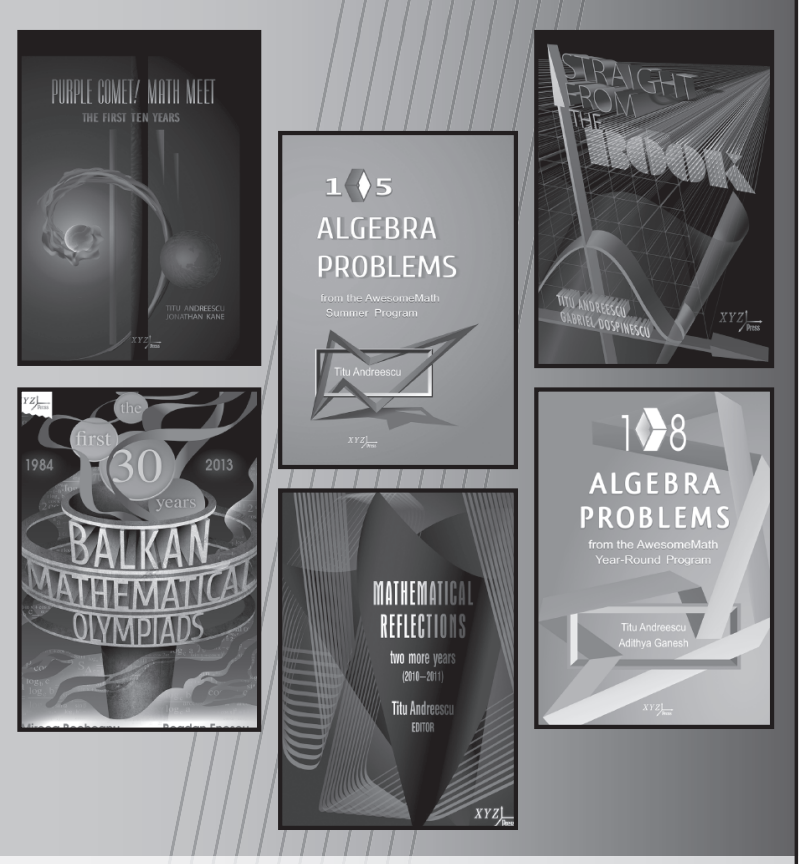

For more information on individual books in this series visit: www.ams.org/bookstore/xyzseries.html in geometry of numbers and discrete geometry, in particular Hilbert's eighteenth problem. He was one of the two plenary speakers at Asiacrypt2012 and was a plenary speaker at the 2012 Annual Meeting of the Chinese Mathematical Society. He received a Chinese National Science Award in 2009, a von Prechtl Medal from TU Vienna in 2008, and an S. S. Chern Prize of the Chinese Mathematical Society in 2007.

\section{Response from Chuanming Zong}

It is a wonderful honor to receive the 2015 Levi L. Conant Prize from the AMS. In February 1991 I took the train from Beijing, through Moscow and Budapest, to Vienna with a dream of making a mathematical career. It took me seven days, as I recall. In April 1993 I obtained a PhD from TU Vienna, under the supervision of Professor Peter M. Gruber and Professor Edmund Hlawka. They were the first to introduce me to Hilbert's eighteenth problem, which was the seed of my scientific work. When I traveled around the world, I met I. M. Gelfand, F. Hirzebruch, J. K. Moser, C. A. Rogers, and K. F. Roth, who had great influence on my mathematical vision and taste. In my mathematical career I have obtained help and support from Wentsun Wu, Yuan Wang, Kung-Ching Chang, Boju Jiang, Christian Buchta, William Chen, Weiyue Ding, Weinan E, Xiaoshan Gao, Richard Gardner, Martin Henk, Jeff Lagarias, David Larman, Peter Mani-Levitska, Yiming Long, Monika Ludwig, Zhiming Ma, Shige Peng, Richard Pollack, Marjorie Senechal, Gang Tian, Xiaoyun Wang, Joerg Wills, Lo Yang, Guenter Ziegler, and many others. To all of the above, to the AMS, and to the selection committee for this award, I owe my deepest gratitude.

\section{About the Prize}

The Conant Prize is awarded annually to recognize an outstanding expository paper published in either the Notices of the AMS or the Bulletin of the $A M S$ in the preceding five years.

Established in 2001, the prize honors the memory of Levi L. Conant (1857-1916), who was a mathematician at Worcester Polytechnic Institute. The prize carries a cash award of US $\$ 1,000$.

The Conant Prize is awarded by the AMS Council acting on the recommendation of a selection committee. For the 2015 prize, the members of the selection committee were John C. Baez, Thomas F. Banchoff, and John F. Oprea.

Previous recipients of the Conant Prize are: Carl Pomerance (2001); Elliott Lieb and Jakob Yngvason (2002); Nicholas Katz and Peter Sarnak (2003); Noam D. Elkies (2004); Allen Knutson and Terence Tao (2005); Ronald M. Solomon (2006); Jeffrey Weeks (2007); J. Brian Conrey, Shlomo Hoory, Nathan Linial, and Avi Wigderson (2008), John W. Morgan (2009), Bryna Kra (2010), David Vogan (2011), Persi Diaconis (2012), John Baez and John Huerta (2013), and Alex Kontorovich (2014). 